\title{
Surface-associated metal catalyst enhances the sorption of perfluorooctanoic acid to multi-walled carbon nanotubes
}

Chengliang $\mathrm{Li}^{1}$, Andreas Schäffer ${ }^{2}$, Jean-Marie Séquaris ${ }^{1}$, Krisztina László ${ }^{3}$, Ajna

Tóth $^{3}$, Etelka Tombácz ${ }^{4}$, Harry Vereecken ${ }^{1}$, Rong $\mathrm{Ji}^{5, *}$, Erwin Klumpp ${ }^{1, *}$

1 Agrosphere Institute (IBG-3), Forschungszentrum Jülich GmbH, D-52425 Jülich, Germany 2 Biology 5-Environmental Biology and Chemodynamics, RWTH Aachen University, D-52056 Aachen, Germany

3 Department of Physical Chemistry and Materials Science, Budapest University of Technology and Economics, Budapest, Hungary

4 Department of Colloid Chemistry, University of Szeged, Aradi Vt. 1, H-6720 Szeged, Hungary

5 State Key Laboratory of Pollution Control and Resource Reuse, School of the Environment, Nanjing University, 163 Xianlin Avenue, 210046 Nanjing, China

*Corresponding Authors:

Dr. Erwin Klumpp, e-mail: e.klumpp@fz-juelich.de; Tel.: (49) 2461-616635; Fax: (49) 2461-612518,

Dr. Rong Ji, e-mail: ji@nju.edu.cn; Tel.: (86) 25-8968 0581 


\section{Abstract}

Perfluorooctanoic acid (PFOA) sorption behaviour of two commercial multi-walled carbon nanotubes (MWCNTs) (C $150 \mathrm{P}$ from Bayer MaterialScience: BA and C-MWNTs from NanoTechLabs Inc.: CP) was investigated from aqueous solution. The BA nanotubes contain $\mathrm{Co} / \mathrm{Mn} / \mathrm{Mg} / \mathrm{Al}$ catalysts both on their outer surface and in the inner bore while $\mathrm{CP}$ contains Fe-based catalyst typically within the tubes. The adsorption isotherms of ${ }^{14} \mathrm{C}$-radiolabeled PFOA were measured by batch experiments and fitted to the Freundlich model $\left(r^{2}>0.92\right) .$. The adsorption affinity and capacity on BA were significantly higher than on CP. Increasing the $\mathrm{pH}$ reduced the adsorption of PFOA due to the electrostatic interaction between the $\mathrm{pH}$-sensitive surface and the adsorbate. Increasing $\mathrm{NaCl}$ concentration lead to the aggregation of the MWCNTs reducing the available surface and thus the adsorption capacity. Removal of the catalyst from the outer surface of BA changed the electrophoretic mobility from positive to negative value also decreasing the adsorbed amount of PFOA. The surface charge of the surface-associated metal catalyst favors the electrostatic sorption of PFOA. Such surface modification may be a promising way to improve the sorption capacity of MWCNTs for pollutants like PFOA and to broaden their potential application in water purification.

Keywords: Perfluorooctanoic acid; multi-walled carbon nanotubes; metal catalyst; sorption 


\section{Introduction}

Perfluorooctanoic acid (PFOA) is a perfluorinated chemical that is gradually becoming the focus of considerable concern due to its resistance to natural degradation [1] and its widespread occurrence in ecological systems [2]. This chemical is inevitably released into environmental compartments with the large-scale production of PFOA and its precursors, such as fluorotelomer alcohols and perfluoroalkyl sulfonamides [3, 4]. The distribution of PFOA in various environmental compartments, such as in air [5], aqueous phase [6,7], snow [8], sediments [9, 10], soils [11], biota [12] and humans [13], has been frequently documented. It is a ubiquitous and very challenging pollutant due to its persistence in the environment. The environmental concentration of PFOA ranges from $0.24 \mathrm{ng} \mathrm{L}^{-1}$ to $11.3 \mu \mathrm{g} \mathrm{L}^{-1}$ in aqueous phase [6, 7]. Generally, the adsorption capacity of PFOA on soil/sediment is low [14]. In order to reduce its risk in the environment, carbon nanotubes (CNTs) are investigated in this paper as potential adsorbents for PFOA.

CNTs consist of graphene sheets rolled into a cylinder [15] and are generally regarded as ideal candidates for widespread applications due to their unique physicochemical properties. Commercial CNTs have potential environmental relevance as both membrane-fixed sorbents and dispersed-phase sorbents [16-20]. In particular, potential environmental applications of CNTs as superior sorbents have been intensively studied for a number of inorganic and organic toxic chemicals [21-29]. Recently, the sorption of PFOA on multi-walled carbon nanotubes (MWCNTs) with different oxygen contents has been investigated in a extended concentration range [30]. MWCNT electrodes were successful used-effective have been prepared and applied in the removal of perfluorochemicals like PFOA by electrochemically 
Chengliang Li, Andreas Schäffer, Jean-Marie Séquaris, Krisztina László, Ajna Tóth, Etelka Tombácz, Harry Vereecken, Rong Ji, Erwin Klumpp Surface-associated metal catalyst enhances the sorption of perfluorooctanoic acid to multi-walled carbon nanotubes Journal of Colloid and Interface Science, 377, 342-346, 2012

assisted adsorption [31]. Thermally treated MWCNTs, possibly due to the removal of disorderly amorphous carbon and certain surface modification, showed an increased sorption capacity of chlorinated compounds [32].

Various sorption mechanisms have been reported on CNTs. The sorption of metallic ions on CNTs is governed by electrostatic interaction [22, 33], while hydrophobic and dispersive interactions play an important role in the sorption of organic compounds $[23,34,35]$. Thus, PFOA sorption on non-functionalized MWCNTs is attributed to hydrophobic interactions [30]. The $\pi-\pi$ interaction between aromatic chemicals and the graphene-like structure of CNTs has also been reported in several studies (e.g. [17, 36]). However, Pan and Xing [37] recently drew the general conclusion that the sorption of organic compounds on CNTs is regulated by multiple mechanisms, such as hydrophobic interactions, $\pi-\pi$ bonds, electrostatic interactions, and hydrogen bonds. Moreover, micropore diffusion into the CNTs can also play a role in the sorption of contaminants [37].

Commercial CNTs vary significantly with respect to catalyst and impurities [38-40]. For the synthesis of CNTs transitional metal catalysts such as Ni, Co, Mo, and Fe are frequently used $[38,41]$. The catalytic metals are mostly in oxide form $[45,51]$. For most commercial CNTs the catalyst is intercalated within the tubes and is not available for the solute [42]. For conductive polymer composites CNTs with surface-associated metal catalyst were developed, $[43,44]$, where the transition metals are in a highly dispersed state along the outer walls as reported by Zhang et al. [45]. The chemical form of the metal in the catalyst, however, was not described: In order to remove the catalyst or its traces from the CNTs, a purification process with strong acids or acid mixtures is usually performed [22, 46, 47]. Nevertheless, 
even after acid washing at high temperature CNTs may contain residual metal catalyst [41], which can be released into the aquatic environment [48]. The role of metal catalyst impurities, which released into the solutions during the sorption, in the sorption of $\mathrm{Pb}$ (II) on $\mathrm{CNTs}$ has recently been investigated [49].

The main objective of our research was to investigate i) the sorption of PFOA on MWCNTs at low concentrations of environmental interest and ii) the influence of surface-associated catalyst on the sorption behavior. To our knowledge, studies on CNTs with surface-associated metal catalyst have not been reported with respect to the sorption of organic pollutants. For this purpose, commercial MWCNTs containing $\mathrm{Co} / \mathrm{Mn} / \mathrm{Al} / \mathrm{Mg}$ catalysts on the outer surface and MWCNTs with Fe-containing catalyst located within the tube are compared. In order to evaluate the role of the surface-associated catalyst, the PFOA sorption after hydrochloric acid $(\mathrm{HCl})$ treatment was also investigated. Non-oxidative $\mathrm{HCl}$ treatment efficiently removes the catalyst from the accessible regions surface of MWCNTs. In addition, the effects of the solution parameters, such as $\mathrm{pH}$ and salinity, on the sorption of PFOA were studied.

\section{Materials and methods}

\subsection{Chemicals}

Non-labeled PFOA (96\% purity) and ${ }^{14} \mathrm{C}$-labeled PFOA (99\% radiochemical purity and $2.04 \times$ $10^{9} \mathrm{~Bq} \mathrm{mmol}^{-1}$ specific activity) were purchased from KMF Laborchemie Handels GmbH (Germany) and Biotrend Chemikalien GmbH (Germany), respectively. The water solubility of PFOA (Molecular weight: 414.07; chemical formula: $\mathrm{C}_{8} \mathrm{HF}_{15} \mathrm{O}_{2}$ ) is $3.4 \mathrm{~g} \mathrm{~L}^{-1}$ at room 
Chengliang Li, Andreas Schäffer, Jean-Marie Séquaris, Krisztina László, Ajna Tóth, Etelka Tombácz, Harry Vereecken, Rong Ji, Erwin Klumpp Surface-associated metal catalyst enhances the sorption of perfluorooctanoic acid to multi-walled carbon nanotubes Journal of Colloid and Interface Science, 377, 342-346, 2012

temperature. The $K_{\mathrm{OW}}$ value of PFOA is unknown [50]. To prepare a stock solution, 1 gram of non-labeled PFOA was weighed into a $1 \mathrm{~L}$ volumetric flask and made up to $1 \mathrm{~L}$ with Milli-Q water. The resistivity of Milli-Q water was greater than $18 \mathrm{M} \Omega \cdot \mathrm{cm}$. The solution was ultrasonicated for 0.5 hour (Sonicator, $50-65 \mathrm{KHz}$ ). Stock solutions were prepared by mixing non-labeled and ${ }^{14} \mathrm{C}$-labeled PFOA solutions and stored at $4{ }^{\circ} \mathrm{C}$ prior to usage.

\subsection{Adsorbents}

MWCNTs of purity $>95 \%$ were purchased from NanoTechLabs Inc. (Yadkinville, NC, USA, C-MWNT) (CP) and Bayer MaterialScience, Germany (Baytubes C 150 P) (BA). According to the Manufacturer's Reports, the MWCNTs were synthesized by the chemical vapor deposition (CVD) method, using Fe nanoparticles and $\mathrm{Co} / \mathrm{Mn} / \mathrm{Mg} / \mathrm{Al}$, respectively, for $\mathrm{CP}$ and BA as the metallic catalyst. The TEM images of BA are shown in Fig S1 in the Supplementary Information. The BA sample also contains metal catalyst on the outer surface in addition to the catalyst in the inner bore of the tubes (http://www.baytubes.com/product_production/baytubes_data.html) [45]. The chemical form of the metal in the catalyst, however, is not specified by the manufacturer. In order to remove the metal impurities from MWCNTs, pristine MWCNTs were mixed with $12.5 \%$ (v/v) $\mathrm{HCl}$ solution (solid to liquid ratio 1: 5000) at ambient temperature. Briefly, the mixtures were ultrasonicated for 10 minutes (Sonicator, 50-65 KHz), stirred overnight, washed with Milli-Q water to neutral $\mathrm{pH}$, filtrated, and then dried at $353 \mathrm{~K}$ in an oven. It has been pointed out that $\mathrm{HCl}$ treatment only removes impurities from the outer surface of MWCNTs [46]. Although intensive ultrasonication in strong oxidative media may lead to cleavage of CNTs into smaller 
fragments [52] our conditions were mild enough not to damage the nanotubes. No changes were observed in the length and diameter distribution of CNTs from the TEM images after purification and/or sonication (not shown here).

Elemental analysis of MWCNTs was performed with inductively coupled plasma mass spectrometry (ICP-MS) (Elan 6000). 30-50 mg of MWCNT was fused with 1 gram of lithium borate at $1323 \mathrm{~K}$, then dissolved in $\mathrm{HNO}_{3}$ solution at ambient temperature and diluted with Milli-Q water to $100 \mathrm{~mL}$. Finally, the concentration of elements in the solution was quantified by ICP-MS. The specific surface area (SSA) of the MWCNTs was determined from $77 \mathrm{~K} \mathrm{~N}_{2}$ adsorption data measured with an AUTOSORB-1 (Quantachrome) apparatus by the multipoint Brunauer-Emmett-Teller (BET) method. Thermogravimetric measurements of MWCNTs were conducted with a simultaneous thermal analyzer 429 (Netzsch, Gerätebau GmbH, Germany). The electrophoretic mobility of the MWCNT suspension (BA: $100 \mathrm{mg} \mathrm{L}^{-1}$ and CP: $250 \mathrm{mg} \mathrm{L}^{-1}$ ) was measured with a Zetasizer Nano series (Malvern) after ultrasonication for 10 minutes (Sonicator, $50-65 \mathrm{KHz}$ ).

\subsection{Batch sorption}

The liquid-phase sorption-desorption experiments were performed in $16 \mathrm{ml}$ volumetric Pyrex glass tubes equipped with Teflon-lined screw caps using a batch technique at $293 \mathrm{~K}$. Pristine or $\mathrm{HCl}$-treated MWCNTs were weighed into the glass tube and pre-wetted with $400 \mathrm{~mL} \mathrm{NaCl}$ $(10 \mathrm{mM})$ electrolyte solution for 24 hours. Aliquots of the pre-wetted suspension and the radioactive PFOA stock solution (concentration: $1 \mathrm{~g} \mathrm{~L}^{-1}$ and $60 \mathrm{mg} \mathrm{L}^{-1}$ ) were then transferred into the tubes with a pipette and a microsyringe, respectively, reaching $100 \mathrm{mg} \mathrm{L}^{-1} \mathrm{BA}$ and 
$250 \mathrm{mg} \mathrm{L}^{-1} \mathrm{CP}$. Initial concentrations of PFOA in the range of $0-50000 \mu \mathrm{g} \mathrm{L}{ }^{-1}$ and $0-400$ $\mu \mathrm{g} \mathrm{L}^{-1}$ were prepared for BA and $\mathrm{CP}$ samples, respectively. The tubes were immediately sealed and mixed at $150 \mathrm{rpm}$ on a horizontal shaker for 48 hours. Preliminary experiments demonstrated that a period of 48 hours is sufficient to reach adsorption equilibrium (Supplementary Information, Fig S2). The solid and liquid phases were separated by centrifugation for 20 minutes at $10,000 \mathrm{~g}$. For the desorption measurements, half of the supernatant was replaced by the electrolyte solution after the completion of the sorption equilibrium experiment. The equilibration time for desorption experiments was also 48 hours. Concentrations of PFOA in all supernatants were quantified by liquid scintillation counting (LSC). MWCNT-free control tests were also performed simultaneously. The adsorbed amount of PFOA was calculated from the concentration difference between the supernatants and the controls because the mass balance for the sorption of PFOA ranged from 97.6\% to 99.5\%. All experiments were performed in triplicate.

Influence of $\mathrm{pH}$ and salinity was investigated using $200 \mu \mathrm{g} \mathrm{L}{ }^{-1} \mathrm{PFOA}$ and $250 \mathrm{mg} \mathrm{L}^{-1} \mathrm{CP}$ or $100 \mathrm{mg} \mathrm{L}^{-1} \mathrm{BA}$. The $\mathrm{pH}$ was adjusted with $1 \mathrm{M} \mathrm{NaOH}$ or $\mathrm{HCl}$ solutions. The salinity was set in the range of 1 to $500 \mathrm{mM}$ with $\mathrm{NaCl}$ solution.

\subsection{Radioactive determination}

Aliquots of the supernatant were mixed with $4 \mathrm{~mL}$ of Insta-Gel Plus scintillation cocktail (USA) in 5-mL vials and counted on a Tri-Carb B 2500 liquid scintillation counter (Packard Bioscience GmbH, Germany) for 10 min (standard deviation $<2 \%$ ).

\subsection{Evaluation of adsorption data}


The Freundlich model (Eq. (1)) was used to fit the sorption data:

$$
C_{s}=K_{f} C_{w}{ }^{1 / n}
$$

where, $C_{s}\left(\mu \mathrm{g} \mathrm{g}^{-1}\right)$ is the amount adsorbed per unit mass of the adsorbent, $C_{w}\left(\mu \mathrm{g} \mathrm{L}^{-1}\right)$ is the equilibrium concentration of the solute, $K_{f}\left(\mu \mathrm{g}^{(1-1 / n)} \mathrm{L}^{1 / n} \mathrm{~g}^{-1}\right)$ is the Freundlich adsorption coefficient, and $n$ (unitless) is an indicator of the nonlinearity of the isotherm.

\section{Results and Discussion}

\subsection{Adsorption isotherms of PFOA}

Preliminary kinetic investigations indicated that the sorption equilibrium could be reached within a few hours (see Fig S2 in Supplementary Information). Adsorption isotherms of PFOA normalized to the surface area of both pristine and HCl-treated MWCNTs are shown in Figure 1a and $b$ at low concentrations. The surface areas of the pristine BA and CP samples were 206 and $111 \mathrm{~m}^{2} \mathrm{~g}^{-1}$, respectively. The $\mathrm{HCl}$ treatment strongly reduced the metal content of the BA, while that of CP decreased only slightly (Table 1). However, a concurrent rise in the specific surface area (approx. 10\%) upon $\mathrm{HCl}$ treatment occurred in the case of both MWCNTs $\left(\mathrm{BA}=231 \mathrm{~m}^{2} \mathrm{~g}^{-1}, \mathrm{CP}=\right.$ $\left.122 \mathrm{~m}^{2} \mathrm{~g}^{-1}\right)$. The small increase of the surface area may be a consequence of the removal of impurities and the additional sonication during the $\mathrm{HCl}$ treatment, which may reduce the aggregate size [52]. In the low concentration range which is of considerable environmental interest the Freundlich model properly described the sorption isotherms $\left(r^{2} \geq 0.92\right.$, Table 2). The insert in Fig. 1a shows the adsorption isotherm of PFOA on pristine BA up to $50000 \mu \mathrm{g} \mathrm{L}^{-1}$. In this extended concentration range the shape of the log-log plot indicates a second adsorption step where the sorption capacities close to those of $\mathrm{Li}$ et al. [30] were obtained. 
In the concentration range investigated (Fig. 1a) the adsorption capacity of PFOA normalized to the surface area was significantly higher on the pristine BA than on $\mathrm{CP}$, indicating that the surface area is not a limiting factor governing the sorption of PFOA on these MWCNTs. The present results also indicate that the higher Fe catalyst content of CP MWCNTs (see Table 1) is of no significance for the sorption in comparison with BA containing $\mathrm{Co} / \mathrm{Mn} / \mathrm{Mg} / \mathrm{Al}$ catalysts. BA adsorbed roughly six times as much PFOA as $\mathrm{CP}$ at
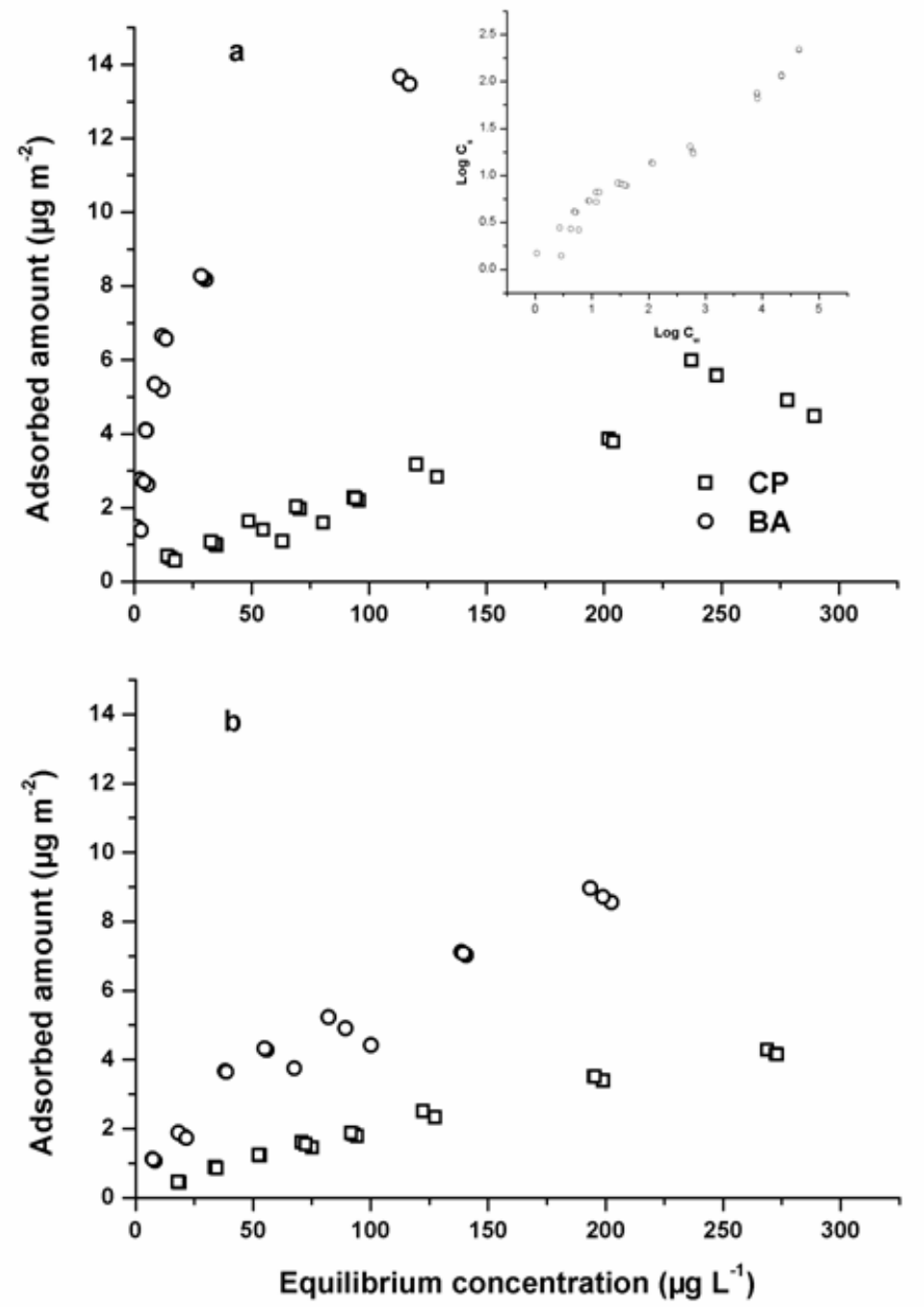

Fig. 1. Adsorption isotherms of PFOA onto (०) BA and ( $\square$ ) CP MWCNTs in $10 \mathrm{mM}$ of $\mathrm{NaCl}$ solution: a) PFOA on pristine MWCNTs, b) PFOA on HCl-treated MWCNTs. The adsorbed amount of PFOA was normalized by surface area. The insert plot shows the sorption 
isotherms of PFOA on pristine BA up to $50000 \mu \mathrm{g} \mathrm{L}{ }^{-1}$.

equilibrium concentration of $100 \mu \mathrm{g} \mathrm{L}^{-1}$. It must be noted that the catalyst within the tubes is generally not available for the solutes from aqueous solutions [42].

The $\mathrm{pH}$ of the supernatants varied in the ranges of 5.8-5.9 and 6.2-6.6 for the pristine $\mathrm{CP}$ and $\mathrm{BA}$, respectively, during the sorption experiments. In these $\mathrm{pH}$ ranges, PFOA is present in an anionic form as its $\mathrm{p} K_{\mathrm{a}}=0.5$ [53]. Variations of the $\mathrm{pH}$ affect not only the ionization of surface functional groups potentially decorating the nanotubes but also more specifically the charge of the surface catalyst. This can be sensitively observed by a microelectrophoretic method. MWCNTs usually have a negative zeta potential under neutral solution conditions [54, 55]. As expected, the electrophoretic mobility (EM) of the pristine $\mathrm{CP}$ was $-1.8 \times 10^{-4} \mathrm{~cm}^{2} \mathrm{~V}^{-1} \mathrm{~s}^{-1}$. On the other hand, in the case of pristine BA a positive zeta potential can be derived from the EM of $+0.9 \times 10^{-4} \mathrm{~cm}^{2} \mathrm{~V}^{-1} \mathrm{~s}^{-1}$. However, the EM of BA MWCNTs was reversed to about $-1.5 \times 10^{-4} \mathrm{~cm}^{2} \mathrm{~V}^{-1} \mathrm{~s}^{-1}$ after $\mathrm{HCl}$ treatment, while that of the $\mathrm{HCl}$-treated $\mathrm{CP}$ was similar to the pristine nanotubes $\left(\mathrm{EM}-1.9 \times 10^{-4} \mathrm{~cm}^{2} \mathrm{~V}^{-1} \mathrm{~s}^{-1}\right)$. This reversal effect in the $\mathrm{EM}$ of the $\mathrm{BA}$ sample after $\mathrm{HCl}$ treatment must be related to the removal of surface-associated fractions of the catalyst. Indeed, a large decrease of metal catalyst content was observed with BA tubes (Table 1) in comparison with the metal content of the $\mathrm{CP}$ tubes which show no substantial change after the $\mathrm{HCl}$ treatment. In the same way, the $\mathrm{EM}$ did not change significantly in the case of $\mathrm{CP}$ tubes. It can be thus hypothesized that the catalyst content on the CP tube surface is low. Regarding the variations of the surface charge demonstrated by the EM and the completely anionic form of PFOA throughout the $\mathrm{pH}$ range 
Chengliang Li, Andreas Schäffer, Jean-Marie Séquaris, Krisztina László, Ajna Tóth, Etelka Tombácz, Harry Vereecken, Rong Ji, Erwin Klumpp Surface-associated metal catalyst enhances the sorption of perfluorooctanoic acid to multi-walled carbon nanotubes Journal of Colloid and Interface Science, 377, 342-346, 2012

investigated it can be also hypothesized that electrostatic interactions play a role in the uptake of PFOA. The variation of the surface charge demonstrated by the EM may be responsible for the different adsorption affinities and capacities. Fig. $1 \mathrm{~b}$ shows that the sorption affinity of PFOA on the HCl-treated BA significantly decreased while only a slight reduction was observed on the $\mathrm{CP}$ sample. The $\mathrm{pH}$ of the aqueous phase during the sorption experiments with $\mathrm{HCl}-$ treated $\mathrm{CP}$ and $\mathrm{BA}$ was in the range of 5.7-6.0 and 6.2-6.7, respectively, i.e. the partial removal of the metal did not result in a significant shift in $\mathrm{pH}$. Therefore, it is assumed that the reduced metal content is responsible for the reduced adsorption affinity of the nanotubes, particularly in the case of BA. The comparison of the EM values associated with the surface charge of the MWCNTS supports this idea. The surface ionizations may arise from the surface acidic groups [56] and oxidized metal catalyst material [45, 57]. The positive EM of BA stems from the positively charged metal oxide particles located on the surface of MWCNTS at neutral solution $\mathrm{pH}$ conditions. Their interaction with the negative perfluorooctanate ions leads to an electrostatic sorption. However, the lack of information about the chemical form of the catalyst limits a further interpretation on atomic level. It also should be mentioned that PFOA desorption from both pristine MWCNTs showed no pronounced hysteresis (Fig. S3 in the Supplementary Information), indicating that the adsorption is reversible.

The sorption results in the low PFOA concentration range investigated here thus support an electrostatic interaction where the ionic properties of the amphiphilic molecule dominate the adsorption behavior. In the absence of surface-associated metal catalyst, i.e., in case of pristine $\mathrm{CP}$ and $\mathrm{HCl}$-treated $\mathrm{CP}$ and $\mathrm{BA}$ the adsorption may be driven by hydrophobic 
interaction and dispersion forces with the carbon skeleton of the MWCNTs [30], resulting in weaker adsorption and lower adsorption capacities.

\subsection{Influence of $\mathrm{pH}$}

Figure 2 shows the influence of $\mathrm{pH}$ on PFOA adsorption to the two pristine nanotubes. In both cases, a decreasing adsorption capacity with increasing $\mathrm{pH}$ value was observed in the $\mathrm{pH}$ range 2-12, which confirms the significance of electrostatic interaction due to the $\mathrm{pH}$-sensitive surface charge. The negative surface charge increases with increasing $\mathrm{pH}$, due to the carboxylic and phenolic groups decorating the surface even of pristine CNTs $[33,56]$.

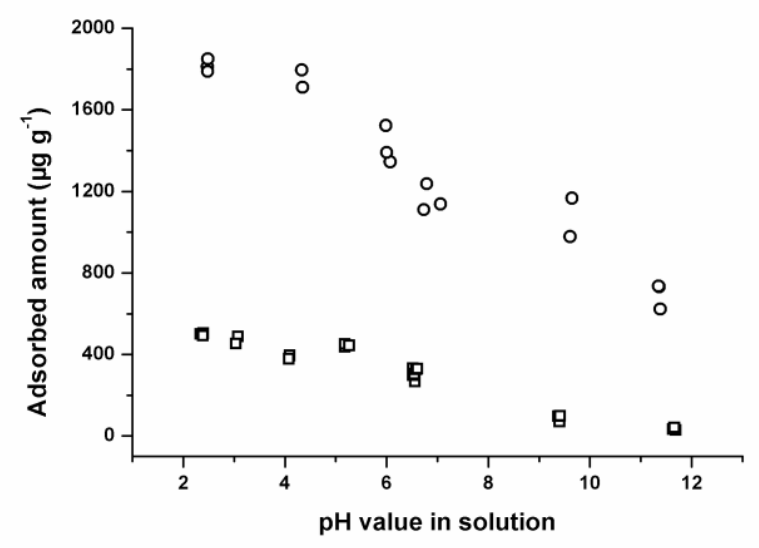

Fig. 2. The influence of $\mathrm{pH}$ on the adsorption of PFOA on the pristine (०) BA and ( $\square$ ) CP MWCNTs. Initial concentration of PFOA is $200 \mu \mathrm{g} \mathrm{L} \mathrm{L}^{-1}$.

However, the presence of a cobalt (Co) oxide phase in BA was reported by Zhang et al.[45], which has a point of zero charge at about $\mathrm{pH} 8[58,59]$. The surface-associated catalyst is thus positively charged in neutral and acidic conditions. As $\mathrm{pH}$ increases the combined effect of the deprotonation of the MWCNTs surface carboxylic groups and the decreasing positive charge of the surface-associated catalyst in the case of BA results in an electrostatic repulsion. 
This essentially limits the sorption of PFOA in the neutral and alkaline $\mathrm{pH}$ ranges. It was also reported that increasing the $\mathrm{pH}$ value decreases the sorption of perfluorinated chemicals on sediments or MWCNTs due to electrostatic repulsion [14]. The further reduction of the adsorption capacity, especially in case of $\mathrm{BA}$ above $\mathrm{pH}$ 6-7, may also be caused by the increasing negative surface charge of the metallic catalyst.

3.3. Influence of salinity (ionic strength)

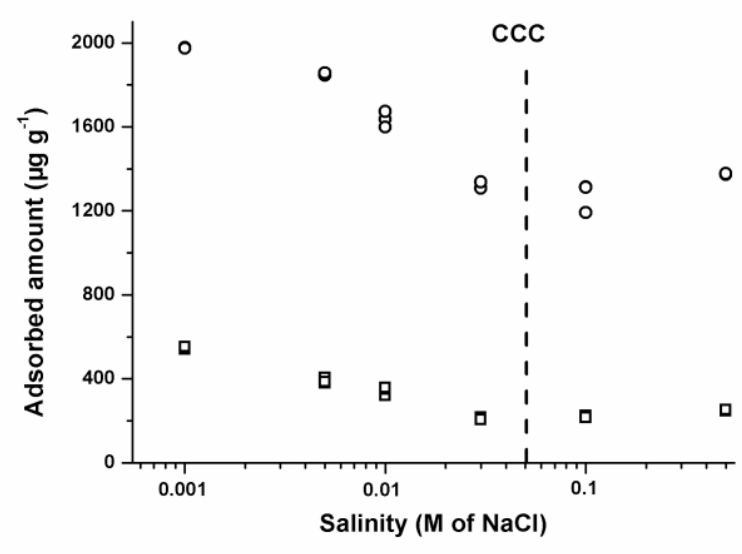

Fig. 3. The influence of salinity on PFOA adsorption on the pristine (०) BA and ( $\square$ ) CP MWCNTs. Initial concentration of PFOA is $200 \mu \mathrm{g} \mathrm{L}{ }^{-1}$.

The influence of salinity on PFOA adsorption on the pristine MWCNTs is shown in Figure 3. In the $0.001-0.05 \mathrm{M} \mathrm{NaCl}$ concentration range the adsorption capacity decreased with increasing salt concentration; above $0.05 \mathrm{M} \mathrm{NaCl}$ the adsorbed amount was almost constant. Chen et al. [47] reported no impact of salinity on the sorption of neutral compounds to single-walled CNTs in solutions containing $0.02-0.1 \mathrm{M} \mathrm{NaNO}_{3}$. This trend is similar to our experience with $\mathrm{NaCl}$ in the same concentration range. On the other hand, an enhanced uptake of polar compounds by activated carbon fiber was detected due to the screening effect of the 
surface charge produced by salt addition [60]. Therefore, it may be hypothesized that the decrease of PFOA adsorption at low salt concentrations can be attributed to the screening of the surface charge by the salt, which induces the aggregation of MWCNTs (Saleh et al., 2008). A critical coagulation concentration $(C C C)$ of about $0.05 \mathrm{M} \mathrm{NaCl}$ (see Fig. S4 in the Supplementary Information) was observed according to the method of Sano et al. [61], who found that the $C C C$ for single-walled CNTs was $0.037 \mathrm{M} \mathrm{NaCl}$. This implies that the influence of the salt concentration on the sorption of PFOA can be attributed to a reduced availability of the MWCNTs surface, as the aggregation offsets the screening effect of the surface charge (Cho et al., 2011). Below the CCC, the gradual decrease of the accessible surface can be related to the slow aggregation kinetics of the dispersed colloidal MWCNTs. Above the $C C C$, a rapid aggregation takes place and the accessible minimum surface remains constant. PFOA only covers about $1 \%$ of the MWCNTs surface in $10 \mathrm{mM} \mathrm{NaCl}$ solution as calculated from the molecular area of PFOA $\left(108 \AA^{2}\right.$ is estimated with the MOLDEN software [62]). A direct influence of PFOA on the MWCNT aggregation is thus improbable. In the case of $\mathrm{BA}$, an adsorption competition of $\mathrm{Cl}^{-}$anions in excess with the deprotonated PFOA $(0.97 \mu \mathrm{M})$ on the positively charged catalyst may also be involved in the observed decreased sorption at $\mathrm{NaCl}$ concentration lower than $0.01 \mathrm{M} \mathrm{NaCl}$ in Figure 3.

\section{Conclusions}

The ad/desorption of PFOA was found completely reversible on pristine BA and CP nanotubes. The sorption was sensitive to the $\mathrm{pH}$ and the salinity of the solution. In the low PFOA concentration range the enhanced sorption capacity of the BA was attributed to the 
presence of $\mathrm{Co} / \mathrm{Mn} / \mathrm{Mg} / \mathrm{Al}$ catalysts on the external surface of the MWCNT.. Although the sorption of PFOA is governed by non-polar interactions at higher concentrations, our results revealed the role played by the carboxylate group of the amphiphilic PFOA molecule in the environmentally relevant low concentration range. Accessible metal catalyst may lead to an enhanced sorption through electrostatic interactions indicating that such surface modification is a promising way to improve the sorption capacity of PFOA on CNTs.

\section{Acknowledgements}

Chengliang Li is grateful for a scholarship from the Deutscher Akademischer Austausch Dienst (DAAD). This study was financed by the National Natural Science Foundation of China (Project Nos.: 20977043 and 20777033).

\section{Supplementary Information}

Supplementary Information associated with this article is attached to the online version.

\section{References}

[1] N. Kudo, Y. Kawashima, J. Toxicol. Sci. 28 (2003) 49.

[2] M. Shoeib, T. Harner, P. Vlahos, Environ. Sci. Technol. 40 (2006) 7577.

[3] T.J. Wallington, M.D. Hurley, J. Xia, D.J. Wuebbles, S. Sillman, A. Ito, J.E. Penner, D.A.

Ellis, J. Martin, S.A. Mabury, O.J. Nielsen, M.P.S. Andersen, Environ. Sci. Technol. 40 (2006)

924.

[4] J.M. Armitage, M. MacLeod, L.T. Cousins, Environ. Sci. Technol. 43 (2009) 1134.

[5] C.J. Mcmurdo, D.A. Ellis, E. Webster, R.D. Christensen, L.K. Reid, Environ. Sci. 
Chengliang Li, Andreas Schäffer, Jean-Marie Séquaris, Krisztina László, Ajna Tóth, Etelka Tombácz, Harry Vereecken, Rong Ji, Erwin Klumpp Surface-associated metal catalyst enhances the sorption of perfluorooctanoic acid to multi-walled carbon nanotubes Journal of Colloid and Interface Science, 377, 342-346, 2012

Technol. 42 (2008) 3969.

[6] M.K. So, S. Taniyasu, N. Yamashita, J.P. Giesy, J. Zheng, Z. Fang, S.H. Im, P.K.S. Lam, Environ. Sci. Technol. 38 (2004) 4056.

[7] M.S. Mclachlan, K.E. Holmstrom, M. Reth, U. Berger, Environ. Sci. Technol. 41 (2007) 7260

[8] C.J. Young, V.I. Furdui, J. Franklin, R.M. Koerner, D.C.G. Muir, S.A. Marbury, Environ. Sci. Technol. 41 (2007) 3455.

[9] C.P. Higgins, R.G. Luty, Environ. Sci. Technol. 41 (2007) 3254.

[10] C.P. Higgins, J.A. Field, C.S. Criddle, R.G. Luthy, Environ. Sci. Technol. 39 (2005) 3946.

[11] M. Ellefson, in: E.D. AR226-1030a030, 3M Company: Maplewood, MN, 2001.

[12] J. Dai, M. Li, Y. Jin, N. Saito, M. Xu, F. Wei, Environ. Sci. Technol. 40 (2006) 5647.

[13] I. Ericson, R. Marti-Cid, M. Nadal, B.V. Bavel, G. Lindstroem, J.L. Domingo, J. Agric. Food Chem. 56 (2008) 1787.

[14] C.P. Higgins, R.G. Luthy, Environ. Sci. Technol. 40 (2006) 7251.

[15] S. Iijima, Nature 354 (1991) 56.

[16] M. Majumder, N. Chopra, B.J. Hinds, J. Am. Chem. Soc. 127 (2005) 9062.

[17] A. Tóth, A. Töröcsik, E. Tombácz, E. Oláh, M. Heggen, C.L. Li, E. Klumpp, E. Geissler, K. László J. Colloid Interface Sci. 364 (2011) 469.

[18] H.-H. Cho, H. Huang, K. Schwab, Langmuir 27 (2011) 12960.

[19] K. Yang, W. Wu, Q. Jing, L. Zhu, Environ. Sci. Technol. 42 (2008).

[20] X. Ren, C. Chen, M. Nagatsu, X. Wang, Chem. Eng. J. 170 (2011) 395.

[21] G. Chen, X. Shan, Y. Wang, Z. Pei, X. Shen, B. Wen, G. Owens, Environ. Sci. Technol. 
$42(2008) 8297$.

[22] C. Lu, H. Chiu, Chem. Eng. Sci. 61 (2006) 1138.

[23] K. Yang, L. Zhu, B. Xing, Environ. Sci. Technol. 40 (2006) 1855.

[24] D. Lin, B. Xing, Environ. Sci. Technol. 42 (2008) 7254.

[25] G.-C. Chen, X.-Q. Shan, Y.-S. Wang, B. Wen, Z.-G. Pei, Y.-N. Xie, T. Liu, J.J. Pignatello, Water Res. 43 (2009) 2409.

[26] C. Lu, Y. Chung, K. Chang, Water Res. 39 (2005) 1183.

[27] Z.Y. Wang, X.D. Yu, B.S. Xing, Environ. Sci. Technol. 44 (2010) 978.

[28] G.D. Sheng, D.D. Shao, X.M. Ren, X.Q. Wang, J.X. Li, Y.X. Chen, X.K. Wang, J. Hazard. Mater. 178 (2010).

[29] K. Pillay, E.M. Cukrowska, N.J. Coville, J. Hazard. Mater. 166 (2009) 1067.

[30] X. Li, H. Zhao, X. Quan, S. Chen, Y. Zhang, H. Yu, J. Hazard. Mater. 186 (2010) 407.

[31] X. Li, S. Chen, X. Quan, Y. Zhang, Environ. Sci. Technol. 45 (2011).

[32] X.M. Ma, D. Anand, X.F. Zhang, S. Talapatra, J. Phys. Chem. C 115 (2011) 4552.

[33] A. Schierz, H. Zanker, Environ. Pollu. 157 (2009) 1088.

[34] S. Gotovac, L. Song, H. Kanoh, K. Kaneko, Colloids Surf. A Physicochem. Eng. Asp. 300 (2007) 117.

[35] S. Gotovac, C.M. Yang, Y. Hattori, K. Takahashi, H. Kanoh, K. Kaneko, J. Colloid Interface Sci. 314 (2007) 18.

[36] W. Chen, L. Duan, D. Zhu, Environ. Sci. Technol. 41 (2007) 8295.

[37] B. Pan, B. Xing, Environ. Sci. Technol. 42 (2008) 9005.

[38] H. Yoshida, T. Shimizu, T. Uchiyama, H. Kohno, Y. Homma, S. Takeda, Nano Lett. 9 
(2009) 3810 .

[39]C. Ge, F. Lao, W. Li, Y. Li, C. Chen, Y. Qiu, X. Mao, B. Li, Z. Chai, Y. Zhao, Anal. Chem. $80(2008) 9426$.

[40] D.L. Plata, P.M. Gschwend, C.M. Reddy, Nanotechnology 19 (2008) 185706.

[41] M. Pumera, Langmuir 23 (2007) 6453.

[42] Y.-Q. Xu, H. Peng, R.H. Hauge, R.E. Smalley, Nano Lett. 5 (2004) 163.

[43] R. Andrews, M.C. Weisenberger, Curr. Opin. Solid ST. M. 8 (2004) 31.

[44] J.N. Coleman, U. Khan, W.J. Blau, Y.K. Gun'ko, Carbon 44 (2006) 1624.

[45] J. Zhang, M. Comotti, F. Schueth, R. Schloegl, D.S. Su, Chem. Commun. 38 (2007) 1916.

[46] V. Datsyuk, M. Kalyva, K. Papagelis, J. Parthenios, D. Tasis, A. Siokou, I. Kallitsis, C. Galiotis, Carbon 46 (2008) 833.

[47] J. Chen, W. Chen, D. Zhu, Environ. Sci. Technol. 42 (2008) 7225.

[48] M.S. Hull, A.J. Kennedy, J.A. Steevens, A.J. Bednar, J.C.A. Weiss, P.J. Vikesland, Environ. Sci. Technol. 43 (2009) 4169.

[49] X. Tian, S. Zhou, Z. Zhang, X. He, M. Yu, D. Lin, Environ. Sci. Technol. 44 (2010) 8144.

[50] K. Prevedouros, I.T. Cousins, R.C. Buck, S.H. Koryeniowski, Environ. Sci. Technol. 40 (2006) 32 .

[51] N.B. Saleh, L.D. Pfefferle, M. Elimelech, Environ. Sci. Technol. 42 (2008) 7963.

[52] E. Farkas, M.E. Anderson, Z.H. Chen, A.G. Rinzler, Chem. Phys. Lett. 363 (2002) 111.

[53] K.U. Goss, Environ. Sci. Technol. 42 (2008) 456. 
[54] J.-P. Tessonnier, D. Rosenthal, T.W. Hansen, C. Hess, M.E. Schuster, R. Blume, F. Girgsdies, N. Pfaender, O. Timpe, D.S. Su, R. Schloegl, Carbon 47 (2009) 1779.

[55] M.L. Usrey, N. Nair, D.E. Agnew, C.F. Pina, M.S. Strano, Langmuir 23 (2007) 7768.

[56] H. Hu, P. Bhowmik, B. Zhao, M.A. Hamon, M.E. Itkis, R.C. Haddon, Chem. Phys. Lett. $345(2001) 25$.

[57] C.-M. Yang, H. Kanoh, K. Kaneko, M. Yudasaka, S. Iijima, J. Phys. Chem. B 106 (2002) 8994.

[58] D. Vickers, L.A. Archer, T. Floyd-Smith, Colloid. Surface. A. 348 (2009) 39.

[59] S. Ardizzone, G. Spinolo, S. Trasatti, Electrochimica Acta 40 (1995) 2683.

[60] M.A. Fontecha-Cámara, A.V. López-Ramón, M.A. Álvarez-Merino, C. Moreno-Catilla, Langmuir 23 (2007) 1242.

[61] M. Sano, J. Okamura, S. Shinkai, Langmuir 17 (2001) 7172.

[62] G. Schaftenaar, J.H. Noordik, J. Compt.-Aided Mol. Design 14 (2000) 123. 